\title{
Lymphotoxin beta receptor is associated with regulation of microRNAs expression and nuclear factor-kappa B activation in lipopolysaccharides (LPS)-stimulated vascular smooth muscle cells
}

\author{
Xiao Ling", Mei Wen", Zezhou Xiao, Zhiwen Luo, Jiawei Zhuang, Qianqin Li, Songlin Du, Shaoyi Zheng, \\ Peng Zhu \\ Department of Cardiovascular Surgery, Nanfang Hospital, Southern Medical University, Guangzhou 510515, China \\ Contributions: (I) Conception and design: P Zhu, S Zheng; (II) Administrative support: None; (III) Provision of study materials or patients: X \\ Ling, M Wen; (IV) Collection and assembly of data: Z Xiao, Z Luo, J Zhuang, Q Li; (V) Data analysis and interpretation: X Ling, M Wen, S Du; \\ (VI) Manuscript writing: All authors; (VII) Final approval of manuscript: All authors. \\ \#These authors contributed equally to this work. \\ Correspondence to: Peng Zhu, MD. Department of Cardiovascular Surgery, Nanfang Hospital, Southern Medical University, 1838 North Guangzhou \\ Avenue, Guangzhou 510515, China. Email: doctff@smu.edu.cn.
}

\begin{abstract}
Background: The aim of present study is to investigate the role of lymphotoxin beta receptor (Lt $\beta r)$ in lipopolysaccharides (LPS)-induced inflammation in vascular smooth muscle cells (VSMCs) and whether its effects are mediated by modulating microRNAs (miRNAs) and nuclear factor-kappa B (NF-кB).

Methods: Mouse aortic smooth muscle cell (SMC) line (MOVAS cells) were transduced with short hairpin $\mathrm{Lt} \beta \mathrm{r}(\operatorname{shLt} \beta \mathrm{r})$ and mRNA and protein expression level of Lt $\beta \mathrm{r}$ were measured by qPCR and Western blot in shLt $\beta r$-transduced cells. Lentiviral vector-transduced (control) and lentiviral vector/shLt $\beta r$-transduced MOVAS cells were stimulated with LPS $(1 \mu \mathrm{g} / \mathrm{mL})$ for 0,16 , or $24 \mathrm{~h}$. Then the mRNA and protein levels of Lt $\beta$ r, interleukin-18 (IL-18), p-p65, p65 and vascular cell adhesion molecule 1 (VCAM-1) were measured by real-time quantitative polymerase chain reaction (qPCR), Western blot and enzyme-linked immunosorbent assay (ELISA). Different miRNAs expression in LPS-stimulated normal and shLt $\beta r$-transduced cells were detected by small RNA sequencing (smRNA-seq).

Results: The mRNA and protein expression of $\mathrm{Lt} \beta \mathrm{r}$ was significantly downregulated in shLt $\beta \mathrm{r}$-transduced cells. LPS-increased the mRNA and protein levels of Lt $\beta r$, IL-18, p-p65 and VCAM-1 in were attenuated by shLt $\beta \mathrm{r}$ transducing compared with LPS-stimulated control group. Moreover, LPS treatment induced 10 upregulated and 64 downregulated miRNAs in shLt $\beta$ r-transduced cells compared with control cells. Moreover, miR-146b-5p and miR-27a-5p levels were significantly decreased in shLt $\beta r$-transduced cells.

Conclusions: Our results show for the first time that the role of Lt $\beta \mathrm{r}$ in regulating inflammatory response in LPS-stimulated VSMCs via modulating miRNAs and NF- $\kappa$ B pathway. Our findings might provide valuable information with respect to better understanding in the treatment of cardiovascular diseases, such as atherosclerosis.
\end{abstract}

Keywords: Lymphotoxin beta receptor $(\mathrm{Lt} \beta \mathrm{r})$; nuclear factor-kappa B (NF- $\kappa \mathrm{B})$; miRNAs; inflammation; vascular smooth muscle cells (VSMCs)

Submitted Sep 28, 2019. Accepted for publication Feb 09, 2020.

doi: 10.21037/apm.2020.03.20

View this article at: http://dx.doi.org/10.21037/apm.2020.03.20 


\section{Introduction}

Vascular inflammation and remodeling are important pathological features of atherosclerosis and vascular smooth muscle cells (VSMCs) play an important role in vascular inflammation and remodeling (1). VSMCs highly express toll-like receptor 4 (TLR4) and can be activated by lipopolysaccharides (LPS). Activated VSMCs can act like immune cells to synthesize and secrete inflammatory mediators to regulate the inflammatory responses which promote the development of vascular inflammation $(1,2)$. And pathophysiological inflammatory processes in VSMCs are mediated by multiple molecules such as inflammatory cytokines and chemokines, adhesion molecules, transcription factors, and miRNAs $(3,4)$.

Increased cytokines [interleukin-8 (IL-8), IL-18, monocyte chemoattractant protein (MCP-1)], adhesion molecules [intercellular cell adhesion molecule-1 (ICAM-1) and vascular cell adhesion molecule 1 (VCAM-1)] are demonstrated to play an important role in the development of atherosclerosis and their expressions are regulated

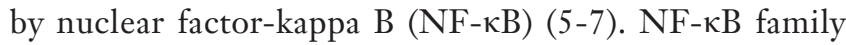
consists of five members: p65 (or RelA), RelB, c-Rel, p50

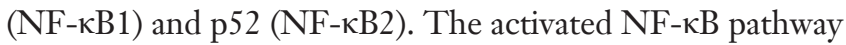
can be divided into classical and non-classical pathway.

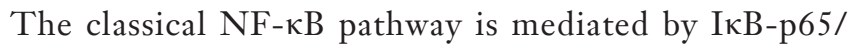
p50 which regulates expression of most proinflammatory genes, including E-selectin, ICAM-1, VCAM-1 and IL-18. While the non-classical NF- $\kappa \mathrm{B}$ pathway is mediated by $\mathrm{NF}-\kappa \mathrm{B}$-inducing kinase (NIK) and IкB kinase $\alpha$ (IKK $\alpha$ )dependent phosphorylation of $\mathrm{p} 52 / \mathrm{p} 100$ which results in the transcription of pro-inflammatory chemokines $(8,9)$. The best studied stimuli to induce classical NF- $\kappa B$ pathway are TNF and LPS, and both classical and non-classical NF$\kappa \mathrm{B}$ pathway can be induced by the activated lymphotoxin beta receptor $(\mathrm{Lt} \beta \mathrm{r})(10,11)$. $\mathrm{Lt} \beta \mathrm{r}$ is a member of the tumor necrosis factor receptor superfamily and constitutively expresses on a wide variety of cells including VSMCs $(12,13)$. $\mathrm{Lt} \beta \mathrm{r}$ has been identified as a key mediator in multiple physiological and pathological processes by interacting with NF- $\kappa B$ (14). Moreover, VSMCs-Lt $\beta r$ has been reported to protect against atherosclerosis by maintaining structure, cellularity and size of artery tertiary lymphoid organs in mice (13). However, how $\mathrm{Lt} \beta \mathrm{r}$ regulates NF- $\kappa \mathrm{B}$ signaling pathway in LPS-stimulated smooth muscle cells (SMCs) is not completely known.

Besides, dysregulation of microRNAs (miRNAs) expression is demonstrated to modulate inflammatory responses by interfering NF- $\mathrm{NB}$ signaling pathway (15) and is associated with many diseases such as atherosclerosis, cancers and diabetes $(7,16)$. miRNAs are a class of highlyconserved, non-coding RNAs of 18-25 nucleotides in length. miRNAs interact with their targets genes and mediate indispensable and negative regulators of gene expression at the post-transcription level (17).

Therefore, in this study, we investigated whether $\mathrm{Lt} \beta \mathrm{r}$ regulated LPS-induced inflammation in VSMCs by modulating miRNAs and NF- $\kappa \mathrm{B}$ activation which might offer new targets for treatment of cardiovascular diseases, such as atherosclerosis.

\section{Methods}

\section{Reagents and antibodies}

Mouse IL-18 ELISA kit was bought from Cusabio biotech. Primary antibodies: polyclonal rabbit anti-Lt $\beta \mathrm{r}$ was from Abcam (catalog number: ab70063), rabbit anti-phosphorNF-кB p65 (p-p65, Ser536, catalog number: 3033) and rabbit anti-p65 (catalog number: 8242) were from Cell Signaling Technology and monoclonal mouse anti-GAPDH (catalog number: HC301) TransGen biotech. Second antibodies: peroxidase-affinipure goat anti-rabbit IgG $(\mathrm{H}+\mathrm{L})$ (catalog number: 101-035-003) and peroxidase-affinipure goat anti-mouse IgG $(\mathrm{H}+\mathrm{L})$ (catalog number: 115-545-166) from Jackson ImmunoResearch. LPS were bought from Sigma.

\section{Cell culture and treatments}

Mouse aortic SMC line (MOVAS cells) was bought from ATCC. Cells were cultured in high-glucose Dulbecco's Modified Eagle Medium (DMEM) supplemented with 10\% fetal bovine serum (FBS) at $37^{\circ} \mathrm{C}$ in a humidified, $5 \% \mathrm{CO}_{2}$ atmosphere. Normal MOVAS cells or shLt $\beta r$ transduced MOVAS cells were treated with or without LPS $(1 \mu \mathrm{g} / \mathrm{mL})$ for 16 or $24 \mathrm{~h}$ in $5 \%$ FBS medium. Each treatment was triplicated with different passage of MOVAS cells.

\section{Enzyme-linked immunosorbent assay (ELISA)}

After confluence, cells were treated with or without LPS $(1 \mu \mathrm{g} / \mathrm{mL})$ for 16 or $24 \mathrm{~h}$ in $5 \%$ FBS medium. Then the medium was centrifuged for 15 minutes at $1,000 \mathrm{~g}$, $4{ }^{\circ} \mathrm{C}$ to remove particulates. The medium was stored at $-20{ }^{\circ} \mathrm{C}$ until the assay started. The level of IL-18 in 
medium was determined by using ELISA kit according to the manufacturer's instruction. The concentration was calculated according to the absorbance of the samples and the standard curve.

\section{Real-time quantitative polymerase chain reaction (qPCR)}

Total RNA was extracted from MOVAS and shLt $\beta \mathrm{r}-$ MOVAS cells using TRIzol reagent and reversely transcribed into cDNA with GoTap ${ }^{\circledR}$ RT Master Mix according to the manufacturer's protocol. qPCR was performed using a $20 \mu \mathrm{L}$ reaction containing $10 \mu \mathrm{L}$ GoTap ${ }^{\circledR}$ RT Master Mix, $1 \mu \mathrm{L} 10 \mu \mathrm{m}$ forward primer, $1 \mu \mathrm{L} 10 \mu \mathrm{m}$ reverse primer $1.5 \mu \mathrm{L}$ template DNA and $6.5 \mu \mathrm{L}$ nucleasefree water. The instrumentation used was the Bio-Rad, MiniOption real-time PCR machine. Initial denaturation was at $95^{\circ} \mathrm{C}$ for 120 seconds followed by 40 cycles of $95^{\circ} \mathrm{C}$ denaturation for 15 seconds, and $60^{\circ} \mathrm{C}$ annealing and extension for 30 seconds. Positive and negative controls were tested in each run.

The specific primers used in this study were as follows: Mus-Lt $\beta r$ : 5'-GCAGCCAAGACACGGT TTG-3' (forward), Mus-Lt $\beta r$ : 5'-AGCCCAGCACAAT GTCACAG-3' (reverse); Mus-Vcam1: 5'-CACAAGTT GGGGATTCGGTT-3' (forward), Mus-Vcam1: 5'-CCTC AAAACCCACAGAGCTCA-3' (reverse); Mus-I118: 5'-TCCTTTGAGGAAATGGATCCAC-3' (forward), Mus-Il18: 5' -TGGCAAGCAAGAAAGTGTCCT-3' (reverse); GAPDH: 5'-GTATGACTCTACCCACG GCAAGT-3' (forward), 5'-TTCCCGTTGATGACCAG CTT-3' (Reverse).

The cycle threshold (Ct) obtained for target gene expression was normalized to GAPDH. Efficiency of reaction was calculated from the slope using the formula $\mathrm{E}=10^{(-1 / \text { slope })}$. The relative expression levels of target genes (normalized to that of GAPDH) were calculated using the $2^{-\Delta \Delta C t}$ method (18). All qPCR experiments were repeated three times.

\section{Western blot analysis}

After treatment, cells were washed twice with ice-cold PBS, lysed with RIPA lysis buffer, centrifuged and quantified with a Bradford protein assay kit according to the manufacturer's instruction. Proteins were separated by $10 \%$ SDS-PAGE and then transferred to PVDF membranes. Membranes were blocked in $5 \%$ nonfat milk/TBST for $1 \mathrm{~h}$ at room temperature and then incubated with primary antibodies [polyclonal rabbit anti-Lt $\beta \mathrm{r}(1: 1,000)$, rabbit anti-p-p65 $(1: 1,000)$, rabbit anti-p65 (1:1,000) and monoclonal mouse anti-GAPDH $(1: 5,000)]$ overnight at $4{ }^{\circ} \mathrm{C}$. After three times washes, membranes were incubated with second antibodies [goat anti-rabbit IgG $(\mathrm{H}+\mathrm{L})(1: 3,000)$ and goat anti-mouse $\operatorname{IgG}(\mathrm{H}+\mathrm{L})(1: 3,000)]$ for $1 \mathrm{~h}$, washed three times and subsequently visualized using ECL kit.

\section{MOVAS transduction with lentiviral short hairpin Ltßr (shLtßr) vectors}

RNA interference (RNAi) is widely used in gene knockdown analysis and as a tool to investigate the function of specific genes or proteins. In order to investigate the role of Lt $\beta \mathrm{r}$ in VSMCs inflammation, the expression of $\mathrm{Lt} \beta \mathrm{r}$ was silenced in MOVAS as previously described. The stable complex of shLt $\beta r$ (interference sequence: GCCAAGACACGGTTTGCAA) and lentiviral vector (LV3-M-Lt $\beta r$-shRNA1) was constructed by GenePharma. The control group was transfected with the complex of non-targeting controls-shRNA (validated not to affect any gene) and lentiviral vector. Before transduction, $1 \times 10^{5}$ cells/ well were cultured in 24-well plates for 18-24 h. When cells were about $2 \times 10^{5}$ cells/well, cells were incubated with complex and $6 \mu \mathrm{g} / \mathrm{mL}$ polybrene in $2 \mathrm{~mL}$ medium without FBS for $4 \mathrm{~h}$. Then $2 \mathrm{~mL}$ fresh medium was added into each well for diluting the concentration of polybrene. After 12-24 h transduction, the medium was then changed into normal medium and cultured for $48 \mathrm{~h}$. Seventy-two hours after transduction, the cells were stimulated with LPS as described above and then harvested for Western blotting or total RNA isolation.

\section{Small RNA sequencing (smRNA-seq) and data analysis}

smRNA-seq was performed as previously described (18). Briefly, total RNA was isolated as above mentioned. Then RNA samples were first DNase-treated and assessed for total quality using Agilent 2100 Bioanalyzer, followed by 2 rounds of polyadenylate positive (poly A+) selection and conversion to cDNA. RNA sequencing was performed on the Illumina HiSeq 2500 using the latest versions of sequencing reagents. TargetScan 7.0 was used to predict target genes.

\section{Statistical analysis}

The data were presented as the means \pm SD. The 
A

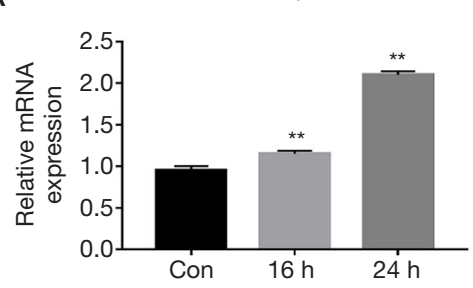

$\mathrm{D}$

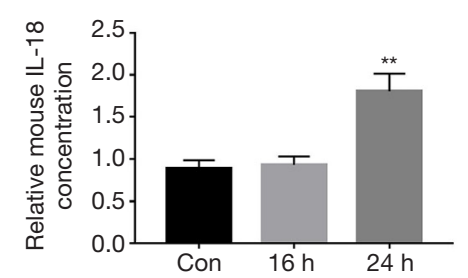

$\mathrm{F}$

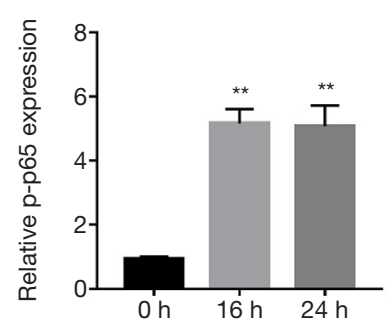

B

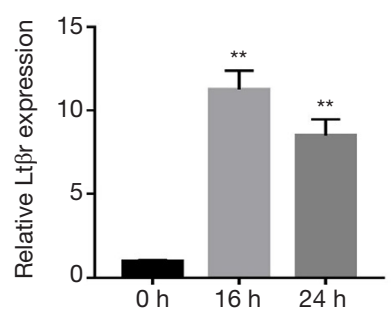

$E$
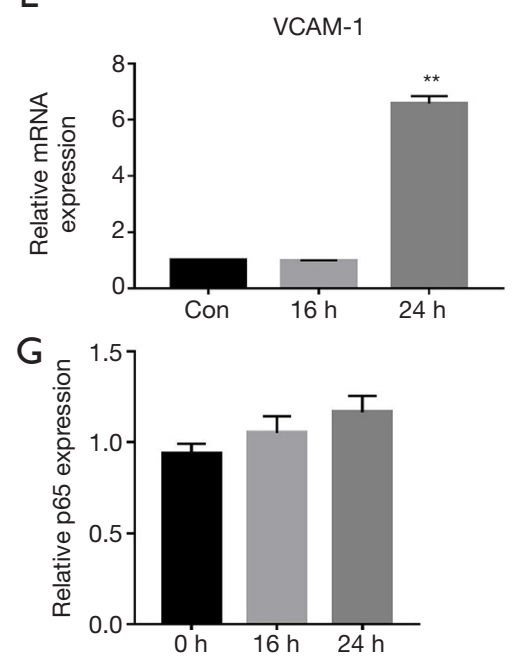

C

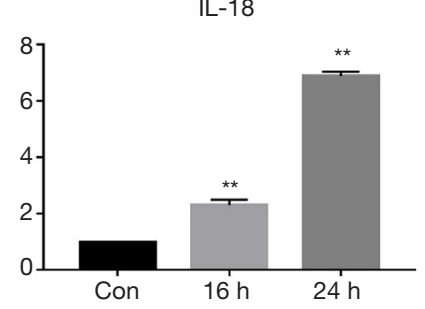

Figure 1 LPS stimulation increased gene and/or protein expression of IL-18, VCAM-1, Lt $\beta r$ and p-p65. (A,B) mRNA and protein expression of Lt $\beta r$ was detected by qPCR and Western blot in 0, 16 and 24 h LPS stimulation group; (C,D) mRNA and protein expression of IL-18 was detected by qPCR and ELISA in 0,16 and 24 h LPS stimulation group; (E) mRNA expression of VCAM-1; (F,G) protein expression of p-p65 and p65; $(\mathrm{H})$ representative Western blot of Ltßr, p-p65 and p65. GAPDH acts as loading control. The experiments were independently repeated three times $(\mathrm{n}=3) .{ }^{* *}, \mathrm{P}<0.01 v s$. control. VCAM-1, vascular cell adhesion molecule $1 ; \mathrm{Lt} \beta \mathrm{r}$, lymphotoxin beta receptor; LPS, lipopolysaccharides.

measurement data were compared between the two groups with Student's $t$-test. The statistical analyses were conducted with SPSS 24.0 software. $\mathrm{P}<0.05$ or $\mathrm{P}<0.01$ was considered statistically significant.

\section{Results}

\section{LPS induced gene and/or protein expression of IL-18, VCAM-1, Ltקr and p-p65 in MOVAS cells}

Normal MOVAS cells were first treated with or without LPS $(1 \mu \mathrm{g} / \mathrm{mL})$ for 0,16 or $24 \mathrm{~h}$. Then the gene and protein levels of Lt $\beta r$, IL-18, VCAM-1 and p-p65 were analyzed by qPCR, Western blot and ELISA. Lt $\beta$ r, IL-18, VCAM-1 and p-p65 were expressed on unstimulated cells with relative low levels (Figure 1A,B,C,D,E,F,G). mRNA and protein expression of $\mathrm{Lt} \beta \mathrm{r}$ were significantly $(\mathrm{P}<0.01)$ increased by 16 and $24 \mathrm{~h}$ LPS stimulation (Figure 1A,B), mRNA expression of IL-18 was increased by 16 and $24 \mathrm{~h}$ LPS stimulation, while protein level of IL-18 in medium was only increased by $24 \mathrm{~h}$ LPS stimulation (Figure $1 C, D$ ). VCAM-1 mRNA expression was only increased by $24 \mathrm{~h}$ LPS stimulation (Figure 1E). Protein expression of phosphorylated p65 was also significantly $(\mathrm{P}<0.01)$ increased by 16 and $24 \mathrm{~h}$ LPS treatment and non-phosphorylated p65 was not changed by LPS stimulation (Figure 1F,G,H). Therefore, $24 \mathrm{~h}$ stimulation was chosen for the following experiments.

\section{LPS-induced expression of inflammatory factors was attenuated in shLtßr transduced MOVAS cells}

To investigate the interaction between Lt $\beta \mathrm{r}$ and LPSinduced inflammation in MOVAS cells, $\mathrm{Lt} \beta \mathrm{r}$ was silenced 
A

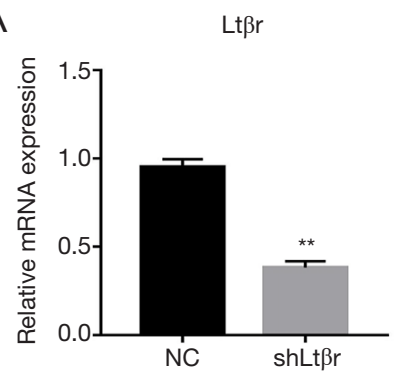

B

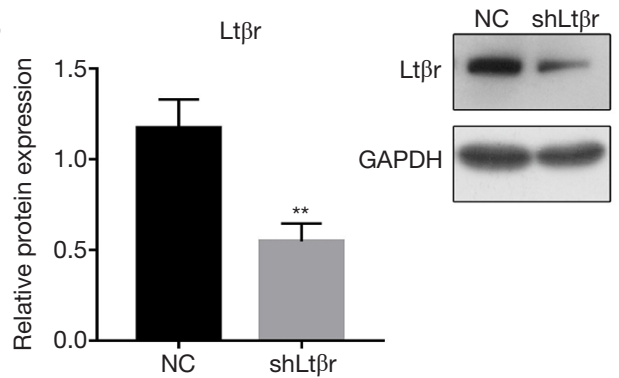

Figure 2 shLt $\beta r$ transduction. (A,B) Lt $\beta r$-specific shRNA depleted gene and protein expression of $\mathrm{Lt} \beta r$. GAPDH immunoblots were used as a loading control. The experiments were independently repeated three times $(\mathrm{n}=3) .{ }^{* *}, \mathrm{P}<0.01 v s$. normal cells. Lt $\beta r$, lymphotoxin beta receptor; shLt $\beta r$, short hairpin Lt $\beta r ; \mathrm{NC}$, negative control.

by using $\mathrm{Lt} \beta \mathrm{r}$-specific shRNA and examined the levels of inflammatory factors including IL-18, VCAM-1 and $\mathrm{p}-\mathrm{p} 65$. We first found that at $72 \mathrm{~h}$ after shLt $\beta \mathrm{r}$ transduction, more than $60 \%$ gene and protein expression of $L t \beta r$ was significantly and stably inhibited compared with the control group (Figure $2 A, B$ ), while cell viability was unaffected (data not shown). Then, we examined the levels of LPS-induced inflammatory factors including Lt $\beta \mathrm{r}$, IL-18, VCAM- 1 and p-p65 in shLt $\beta \mathrm{r}$ transduced MOVAS cell. mRNA and protein levels of $\mathrm{Lt} \beta \mathrm{r}$ were still upregulated by LPS stimulation. However, the levels were significantly lower than in LPSstimulated non-shLt $\beta \mathrm{r}$ MOVAS cells (Figure $3 A, B$ ). LPSinduced IL-18, VCAM-1 and p-p65 expression in shLt $\beta \mathrm{r}$ transduced cells showed similar trend with expression of $\mathrm{Lt} \beta \mathrm{r}$ (Figure 3C,D,E,F,G,H).

\section{Altered endogenous miRNA biogenesis by shLtßr transduction}

As mentioned above, miRNAs played an important role in regulating inflammation. Therefore, whether miRNAs expression was changed by shLt $\beta r$ transduction was determined by smRNA-Seq analysis. LPS-induced different miRNAs expression in only lentiviral vector (without shLt $\beta \mathrm{r}$ sequence)-transduced MOVAS cells and lentiviral vector/shLt $\beta r$-transduced MOVAS cells was compared. With smRNA sequencing analysis, a total of 1,917 miRNAs were identified in the study. Based on a $95 \%$ confidence level, cutoff values of 2-fold for upregulated and downregulated genes were used to define a gene as being differently expressed gene in present study. Through global normalization of the raw data, heatmap data indicated that 10 miRNAs were upregulated and 64 miRNAs were downregulated (Figure 4). And 64 downregulated miRNAs in shLt $\beta \mathrm{r}$ transduced MOVAS cells were listed in Table 1.

\section{$m i R-146 b-5 p$ and $m i R-27 a-5 p$ levels were significantly decreased in shLtßr transduced MOVAS cells}

For further study, miRNAs were chosen based on (I) decreased miRNAs expression in LPS-stimulated shLt $\beta r$ MOVAS cells compared with LPS-stimulated normal cells; (II) upregulated miRNAs expression in inflammation found in previous study. Then the expression level of selected miRNAs in samples was confirmed by qPCR. And results showed that relative miR-146b-5p and miR-27a$5 \mathrm{p}$ expression was significantly downregulated in LPSstimulated shLt $\beta$ r MOVAS cells compared with LPSstimulated normal MOVAS cells (Figure 5).

\section{Discussion}

VSMCs inflammation induced by LPS characterized by increased production of inflammatory cytokines, chemokines, adhesion molecules, transcription factors and changed miRNAs expression which were involved in the development of atherosclerosis and connect reciprocally (3). Therefore, in present report, we conducted experiments to reveal the downstream of $\operatorname{Lt} \beta \mathrm{r}$ and its regulatory effects on miRNAs/NF- $\kappa \mathrm{B}$ signaling pathway and subsequent inflammatory cytokine and adhesion molecule expression in LPS-stimulated VSMCs for the first time.

Firstly, Lt $\beta r$ was found to be expressed on nonstimulated VSMCs and increased by LPS stimulation which suggested its role in regulation of inflammation in VSMCs. In previous study, $\mathrm{Lt} \beta \mathrm{r}$ activation was involved in inflammatory responses which was mediated by activating

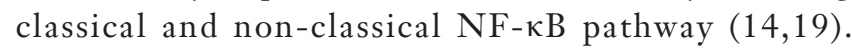


A

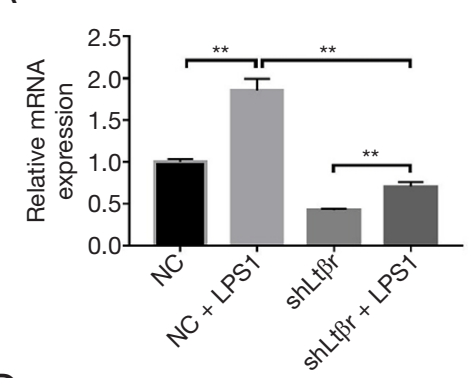

$\mathrm{D}$

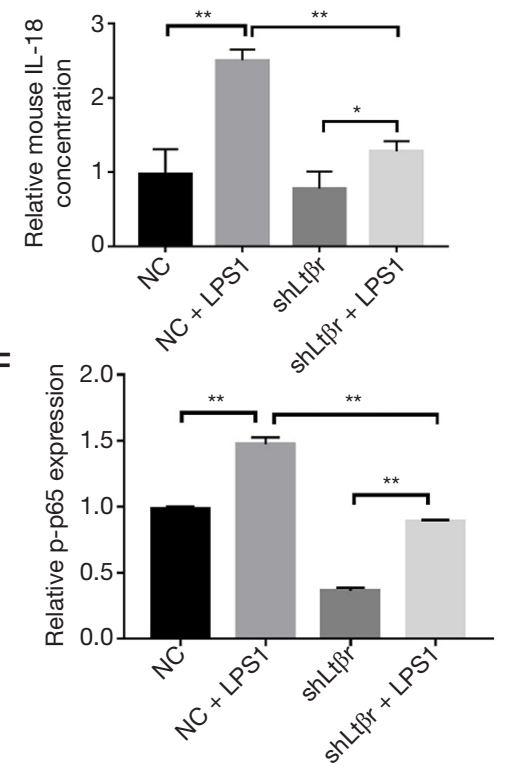

B

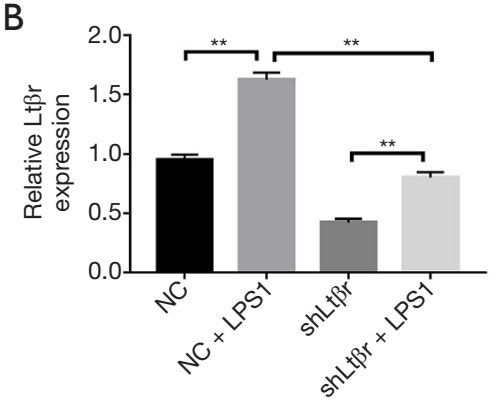

E

G
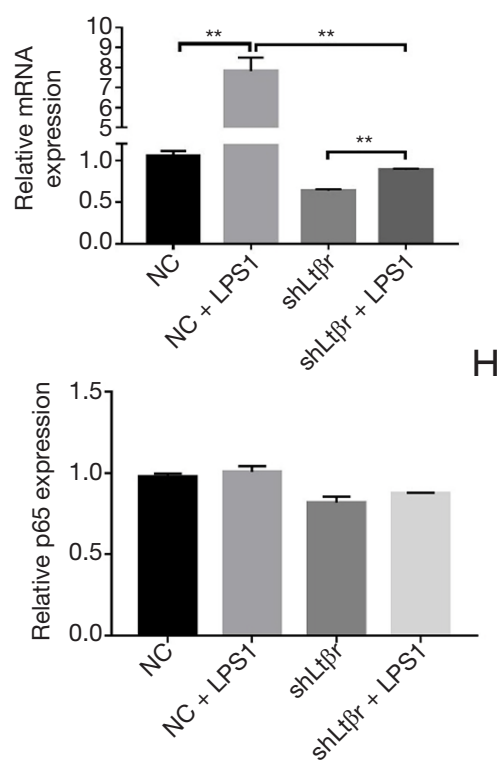
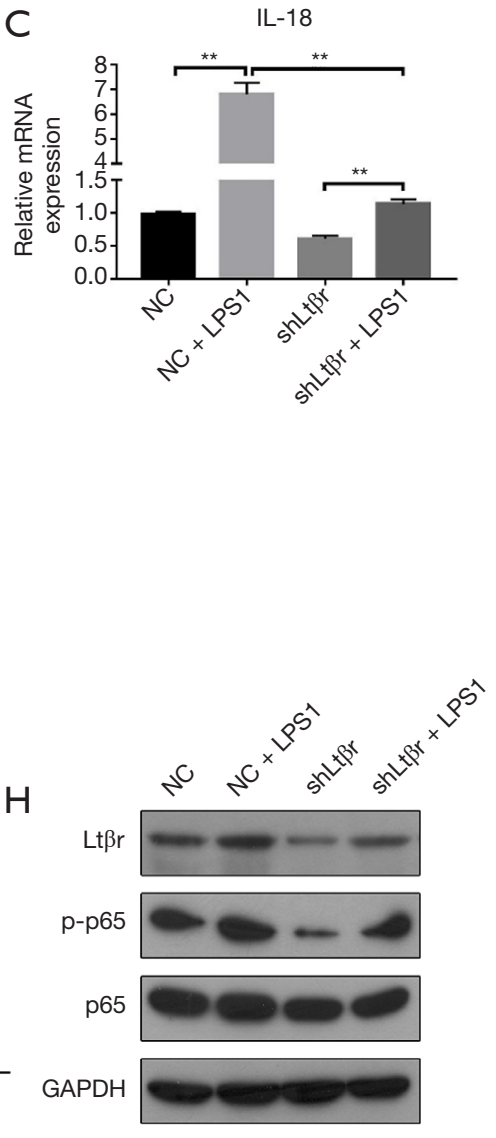

Figure 3 Silencing Lt $\beta r$ reduced LPS-increased expression of IL-18, VCAM-1, Lt $\beta r$ and p-p65. mRNA and protein expression of IL-18, VCAM-1, Lt $\beta r$, p-p65 and p65 was detected in MOVAS cells after transduction with shLt $\beta r$. $(A, B)$ mRNA and protein expression of $L t \beta r$ was detected by qPCR and Western blot; (C,D) mRNA and protein expression of IL-18 was detected by qPCR and ELISA; (E) mRNA expression of VCAM-1; (F,G) protein expression of p-p65 and p65; (H) representative Western blot of Lt $\beta \mathrm{r}$, p-p65 and p65. GAPDH acts as loading control. The experiments were independently repeated three times $(\mathrm{n}=3)$. ${ }^{*}, \mathrm{P}<0.05 ;{ }^{* *}, \mathrm{P}<0.01$. VCAM-1, vascular cell adhesion molecule 1; Lt $\beta$ r, lymphotoxin beta receptor; LPS, lipopolysaccharides.

$\mathrm{NF}-\kappa \mathrm{B}$ is a ubiquitous transcription factor and NF- $\kappa \mathrm{B}$ activation is reported to be crucial for the expression of inflammatory mediators including adhesion molecules (VCAM-1) and cytokines (IL-18) in VSMCs (20-22). VCAM-1 expresses not only on endothelial cells but also SMCs and facilitates the accumulation of transmigrated immune cells within the vascular walls in the development of atherosclerosis (23). IL-18 is a pro-atherogenic cytokine which is highly expressed in SMCs of atherosclerotic lesions. Recombinant IL-18 increases cytokines production and intensify adhesion molecules expression in endothelial cells (6). Lower expression of IL-18 was accompanied by less development of atherosclerosis in mice (20).
Furthermore, elevated levels of plasma IL-18 are associated with the extent of coronary atherosclerosis (24). Therefore, downregulation of VCAM-1 and IL-18 in inflammation will be beneficial for atherosclerosis. In present study, LPSincreased VCAM-1 and IL-18 were restored by shLt $\beta r$ transduction. Furthermore, LPS stimulation-induced the phosphorylation and subsequent translocation of p65 to the nucleus in normal MOVAS cells were also inhibited by shLt $\beta \mathrm{r}$ transduction. All these findings indicated that Lt $\mathrm{Br}-$ regulated VSMCs inflammation including IL-18 production and VCAM-1 expression might be associated with its role in modulating the activation of NF- $\mathrm{kB}$. As we mentioned above, miRNAs were reported to play an important role in 


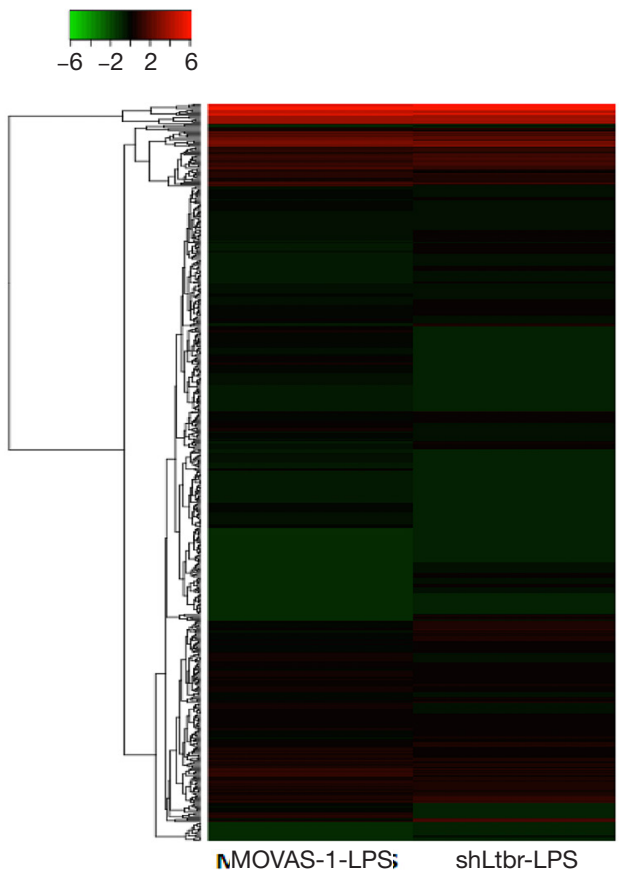

Figure 4 Gene expression levels in shLt $\beta r$ transduced LPS-treated MOVAS cells. Hierarchical clustering of differentially expressed miRNAs in in LPS-treated normal and shLt $\beta$ r transduced MOVAS cells by smRNA-seq. Color intensity values correspond to $\log 2$ of absolute intensity and reach saturation on the heat map at value 6 to preserve dynamic range at lower values. The gene expression profiles on the left were normal MOVAS cells with LPS stimulation and on the right were shLt $\beta \mathrm{r}$ transduced MOVAS cells with LPS stimulation. MOVAS, mouse aortic smooth muscle cell line; shLt $\beta r$, short hairpin lymphotoxin beta receptor; smRNA-seq, small RNA sequencing; LPS, lipopolysaccharides.

regulating the inflammatory responses by modulating gene expression of upstream factors of NF- $\mathrm{\kappa B}$ signaling pathway or directly modulating gene expression of subunits of NF$\kappa \mathrm{B}(25,26)$. Dysregulation of miRNAs was associated with the development of vascular inflammation (27). Therefore, whether miRNAs expression was influenced by shLt $\beta \mathrm{r}$ transduction has also been investigated. miRNAs expression was dysregulated and miR-146b-5p and miR-27a-5b were significantly downregulated in LPS-treated shLt $\beta \mathrm{r}$ cells which were accompanied with inhibited phosphorylation of p65 and NF- $\mathrm{kB}$-mediated inflammatory factors, such as IL18 and VCAM-1.miR-146b-5p acted as an inhibitor of NF$\kappa \mathrm{B}$-mediated inflammation by targeted expression of IL-1 receptor-associated kinase 1 (IRAK1) and TNF receptorassociated factor 6 (TRAF6), upstream regulators of NF$\kappa \mathrm{B}$, in monocytes and endothelial cells. Increased expression of IRAK1 and TRAF6 leaded to more NF- $\kappa$ B p65 DNA binding activity. Altered p65 nuclear translocation leaded to changes in the expression of downstream targets of NF- $\mathrm{BB}$ signaling including VCAM-1 expression $(25,28)$. Besides, miRNAs were also reported to directly regulate $\mathrm{NF}-\kappa \mathrm{B}$ subunit by silencing $\mathrm{NF} \kappa \mathrm{B} 1$ and $\mathrm{p} 65$ to inactivate canonical $\mathrm{NF}-\kappa \mathrm{B}$ signaling (29). miR-146b-5p was found to target $N F \kappa B 1$ gene (data not shown).

Inhibition of miR-27a expression significantly downregulated the expression of TNF $\alpha$ and IL-6 which was associated with altering the expression of both repressors and activators of NF- $\mathrm{BB}$ signaling cascade including RelA gene leading to decreased p65 nuclear translocation in response to proinflammatory stimulant which suggests miR$27 \mathrm{a}-5 \mathrm{p}$ act to regulate the extent of NF- $\kappa \mathrm{B}$ signaling $(26,30)$.

\section{Conclusions}

In summary, two new findings were demonstrated in present study: (I) LPS-induced NF- $\mathrm{KB}$ activation and subsequent pro-inflammatory factors (IL-18 and VCAM-1) expression were inhibited by silencing $\mathrm{Lt} \beta \mathrm{r}$ on MOVAs cells; (II) the expression levels of miR-146b-5p and miR-27a-5p which were reported to modulate the NF- $\kappa \mathrm{B}$ activation were downregulated by shLt $\beta \mathrm{r}$ transduction. We made a conclusion that $\mathrm{Lt} \beta \mathrm{r}$ could regulate VSMCs inflammation in atherosclerosis by modulating miRNAs/NF- $\mathrm{\kappa B} / \mathrm{IL}-18$ VCAM-1 cascade. These findings verify the role of $\mathrm{Lt} \beta \mathrm{r}$ in VSMCs inflammation and provide a potential signaling cascade mediated the effects of $\mathrm{Lt} \beta \mathrm{r}$ which offers new insights into the molecular mechanisms underlying VSMCs 
Table 1 Downregulated miRNAs in shLt $\beta$ r infected MOVAS cells

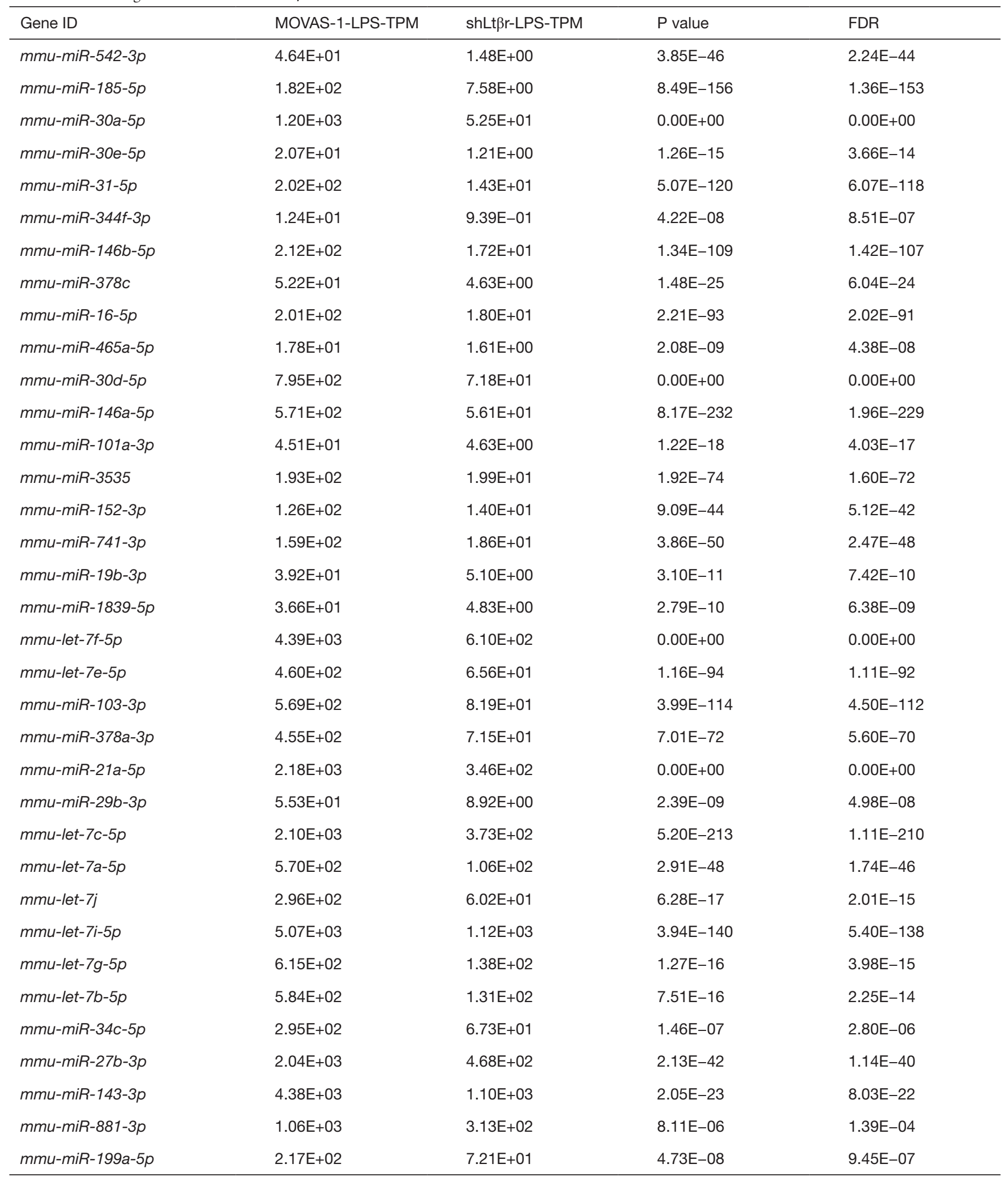

Table 1 (continued) 
Table 1 (continued)

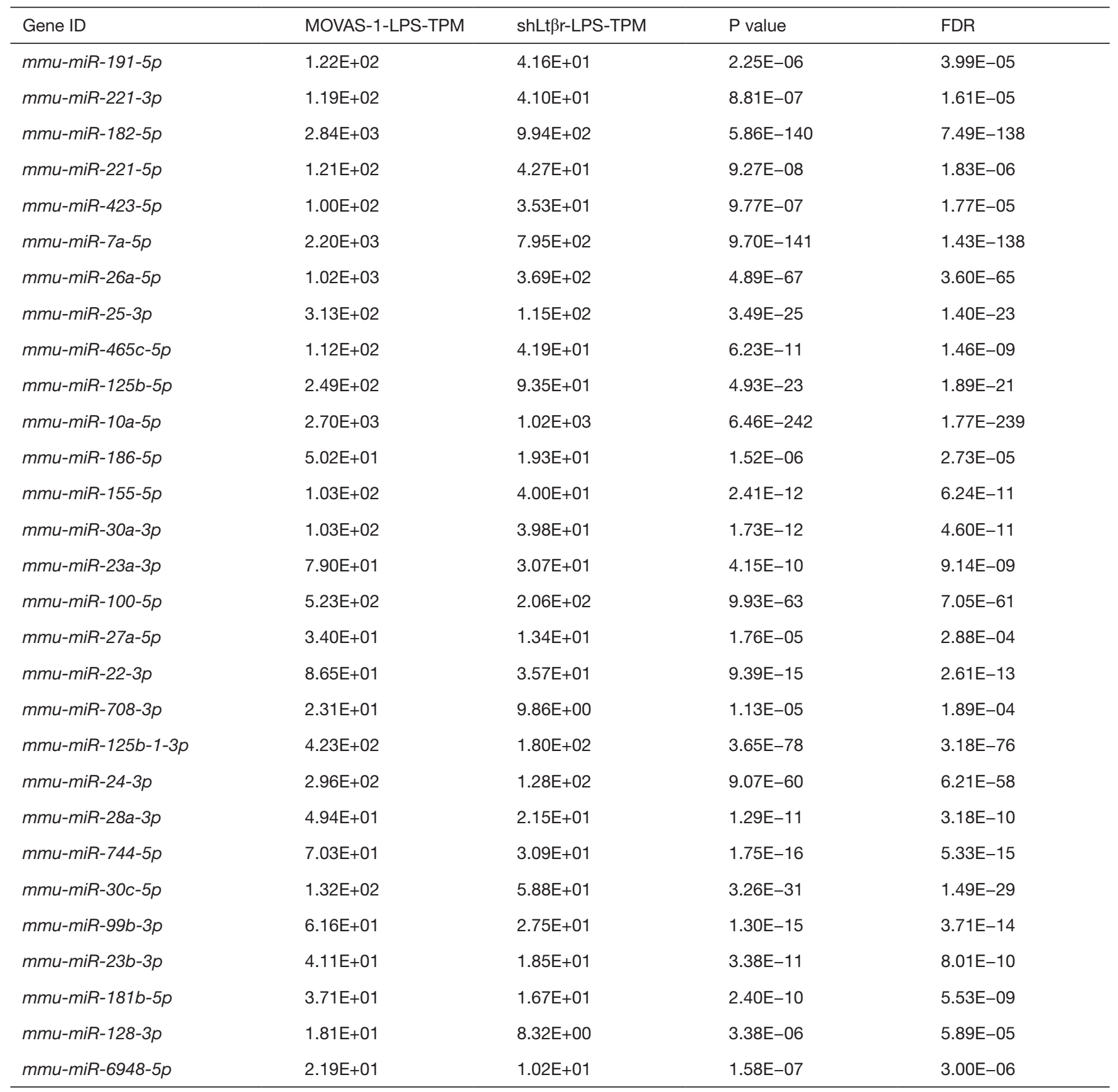

MOVAS, mouse aortic smooth muscle cell line; shLt $\beta r$, short hairpin lymphotoxin beta receptor; LPS, lipopolysaccharides; FDR, false discovery rate. 


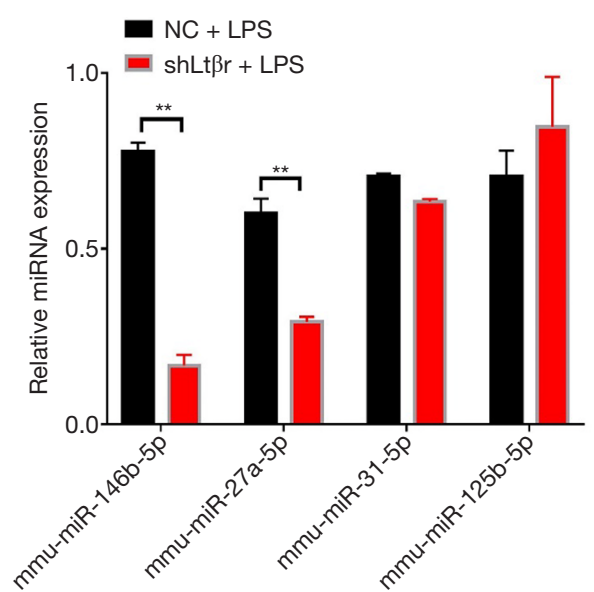

Figure 5 Downregulated miR-146b-5p and miR-27a-5p expression in shLt $\beta r$ MOVAS cells. Relative expression of miR146b-5p and miR-27a-5p were determined by using qPCR analysis in LPS-stimulated normal and shLt $\beta$ r MOVAS cells. ${ }^{* *}, \mathrm{P}<0.01 v s$. control. NC, negative control; LPS, lipopolysaccharides; shLt $\beta$, short hairpin lymphotoxin beta receptor.

dysregulation and pathogenesis of atherosclerosis.

\section{Acknowledgments}

Funding: This work was supported by the President Foundation of Nanfang Hospital, Southern Medical University (grant No. 2017B022). National Natural Science Foundation of China (grant No. 81600321). Science and Technology program of Guangzhou (grant No. 201804010067).

\section{Footnote}

Conflicts of Interest: All authors have completed the ICMJE uniform disclosure form (available at http://dx.doi. org/10.21037/apm.2020.03.20). The authors have no conflicts of interest to declare.

Ethical Statement: The authors are accountable for all aspects of the work in ensuring that questions related to the accuracy or integrity of any part of the work are appropriately investigated and resolved.

Open Access Statement: This is an Open Access article distributed in accordance with the Creative Commons Attribution-NonCommercial-NoDerivs 4.0 International
License (CC BY-NC-ND 4.0), which permits the noncommercial replication and distribution of the article with the strict proviso that no changes or edits are made and the original work is properly cited (including links to both the formal publication through the relevant DOI and the license). See: https://creativecommons.org/licenses/by-nc-nd/4.0/.

\section{References}

1. Chistiakov DA, Orekhov AN, Bobryshev YV. Vascular smooth muscle cell in atherosclerosis. Acta Physiol (Oxf) 2015;214:33-50.

2. Wang P, Han X, Mo B, et al. LPS enhances TLR4 expression and IFNgamma production via the TLR4/IRAK/NFkappaB signaling pathway in rat pulmonary arterial smooth muscle cells. Mol Med Rep 2017;16:3111-6.

3. Varghese JF, Patel R, Yadav UCS. Novel Insights in the Metabolic Syndrome-induced Oxidative Stress and Inflammation-mediated Atherosclerosis. Curr Cardiol Rev 2018;14:4-14.

4. Braun M, Pietsch P, Schrör K, et al. Cellular adhesion molecules on vascular smooth muscle cells. Cardiovasc Res 1999;41:395-401.

5. Li M, Van Esch BCAM, Henricks PAJ, et al. Time and Concentration Dependent Effects of Short Chain Fatty Acids on Lipopolysaccharide- or Tumor Necrosis Factor $\alpha$-Induced Endothelial Activation. Front Pharmacol 2018;9:233.

6. Esfahani M, Saidijam M, Najafi R, et al. The effect of salusin- $\beta$ on expression of pro- and anti-inflammatory cytokines in human umbilical vein endothelial cells (HUVECs). ARYA Atheroscler 2018;14:1-10.

7. Laffont B, Rayner KJ. MicroRNAs in the Pathobiology and Therapy of Atherosclerosis. Can J Cardiol 2017;33:313-24.

8. Mussbacher M, Salzmann M, Brostjan C, et al. Cell Type-Specific Roles of NF-кB Linking Inflammation and Thrombosis. Front Immunol 2019;10:85.

9. Oeckinghaus A, Ghosh S. The NF-kappaB family of transcription factors and its regulation. Cold Spring Harb Perspect Biol 2009;1:a000034.

10. Madge LA, Kluger MS, Orange JS, et al. Lymphotoxinalpha 1 beta 2 and LIGHT induce classical and noncanonical NF-kappa B-dependent proinflammatory gene expression in vascular endothelial cells. J Immunol 2008;180:3467-77.

11. Piao W, Xiong Y, Famulski K, et al. Regulation of T cell 
afferent lymphatic migration by targeting LT $\beta$ R-mediated non-classical NFאB signaling. Nat Commun 2018;9:3020.

12. Lan J, Heneghan AF, Sano Y, et al. Parenteral nutrition impairs lymphotoxin $\beta$ receptor signaling via NF- $\kappa \mathrm{B}$. Ann Surg 2011;253:996-1003.

13. Hu D, Mohanta SK, Yin C, et al. Artery Tertiary Lymphoid Organs Control Aorta Immunity and Protect against Atherosclerosis via Vascular Smooth Muscle Cell Lymphotoxin $\beta$ Receptors. Immunity 2015;42:1100-15.

14. Fernandes MT, Dejardin E, dos Santos NR. Contextdependent roles for lymphotoxin-beta receptor signaling in cancer development. Biochim Biophys Acta 2016;1865:204-19.

15. Yang Y, Wang JK. The functional analysis of MicroRNAs involved in NF-kappaB signaling. Eur Rev Med Pharmacol Sci 2016;20:1764-74.

16. Adlakha YK, Saini N. Brain microRNAs and insights into biological functions and therapeutic potential of brain enriched miRNA-128. Mol Cancer 2014;13:33.

17. Filipowicz $W$, Bhattacharyya SN, Sonenberg N. Mechanisms of post-transcriptional regulation by microRNAs: are the answers in sight? Nat Rev Genet 2008;9:102-14.

18. Pacheco NL, Heaven MR, Holt LM, et al. RNA sequencing and proteomics approaches reveal novel deficits in the cortex of Mecp2-deficient mice, a model for Rett syndrome. Mol Autism 2017;8:56.

19. Dai X, Thiagarajan D, Fang J, et al. SM22 $\alpha$ suppresses cytokine-induced inflammation and the transcription of NF- $\kappa \mathrm{B}$ inducing kinase (Nik) by modulating SRF transcriptional activity in vascular smooth muscle cells. PLoS One 2017;12:e190191.

20. Stein S, Lohmann C, Handschin C, et al. ApoE-/- PGC$1 \alpha-/-$ mice display reduced IL-18 levels and do not develop enhanced atherosclerosis. PLoS One 2010;5:e13539.

21. Kim HJ, Park KG, Yoo EK, et al. Effects of PGC-1alpha on TNF-alpha-induced MCP-1 and VCAM-1 expression and NF-kappaB activation in human aortic smooth muscle and endothelial cells. Antioxid Redox Signal 2007;9:301-7.

22. Li P, Sanz I, O'Keefe RJ, et al. NF-kappa B regulates VCAM-1 expression on fibroblast-like synoviocytes. J Immunol 2000;164:5990-7.

23. Kasper HU, Schmidt A, Roessner A. Expression of the adhesion molecules ICAM, VCAM, and ELAM in the arteriosclerotic plaque. Gen Diagn Pathol 1996;141:289-94.

24. Mysliwska J, Wypych J, Suchanek H. Re: Plasma interleukin (IL)-18 concentrations is elevated in patients with previous myocardial infarction and related to severity of coronary atherosclerosis independently of C-reactive protein and IL-6 [Atherosclerosis 188 (2006) 450-454]. Atherosclerosis 2007;195:415-6.

25. Hulsmans M, Van Dooren E, Mathieu C, et al. Decrease of miR-146b-5p in monocytes during obesity is associated with loss of the anti-inflammatory but not insulin signaling action of adiponectin. PLoS One 2012;7:e32794.

26. Romay MC, Che N, Becker SN, et al. Regulation of NF$\kappa \mathrm{B}$ signaling by oxidized glycerophospholipid and IL$1 \beta$ induced miRs-21-3p and $-27 a-5 p$ in human aortic endothelial cells. J Lipid Res 2015;56:38-50.

27. Ding Y, Sun X, Shan PF. MicroRNAs and Cardiovascular Disease in Diabetes Mellitus. Biomed Res Int 2017;2017:4080364.

28. Echavarria R, Mayaki D, Neel JC, et al. Angiopoietin-1 inhibits toll-like receptor 4 signalling in cultured endothelial cells: role of miR-146b-5p. Cardiovasc Res 2015;106:465-77.

29. Huang T, Kang W, Zhang B, et al. miR-508-3p concordantly silences NFKB1 and RELA to inactivate canonical NF- $\kappa \mathrm{B}$ signaling in gastric carcinogenesis. Mol Cancer 2016;15:9.

30. Wang Z, Ruan Z, Mao Y, et al. miR-27a is up regulated and promotes inflammatory response in sepsis. Cell Immunol 2014;290:190-5.
Cite this article as: Ling $\mathrm{X}$, Wen $M$, Xiao Z, Luo Z, Zhuang J, Li Q, Du S, Zheng S, Zhu P. Lymphotoxin beta receptor is associated with regulation of microRNAs expression and nuclear factor-kappa B activation in lipopolysaccharides (LPS)stimulated vascular smooth muscle cells. Ann Palliat Med 2020;9(3):805-815. doi: 10.21037/apm.2020.03.20 